\title{
The zinc matrix metalloproteinase ZMP-2 increases survival of Caenorhabditis elegans through interference with lipoprotein absorption
}

\author{
Malaika Fischer • Elena Fitzenberger • \\ Rebecca Kull • Michael Boll • Uwe Wenzel
}

Received: 11 April 2014/Accepted: 17 June 2014/Published online: 24 June 2014

(C) Springer-Verlag Berlin Heidelberg 2014

\begin{abstract}
Matrix metalloproteinases are zinc-dependent endopeptidases conserved throughout the animal kingdom which primarily degrade components of the extracellular matrix. In the nematode Caenorhabditis elegans, the zinc matrix metalloproteinase (ZMP-2) was demonstrated to increase resistance versus heat and bacterial pathogens. Here, we show that the survival reducing activities caused by the knockdown of zmp-2 in C. elegans essentially requires the presence of vitellogenin-6, a protein homologous to mammalian apolipoprotein $\mathrm{B}$, and RME-2, a receptor mediating endocytosis of cholesterol particles. Measurements of reactive oxygen species inside and outside $C$. elegans revealed that knockdown of zmp-2 causes a prooxidative extracellular mileu which is a prerequisite for the reduction of survival. Interestingly, RNAi for the foxo transcription factor daf-16 completely prevented those survival reducing effects of $z m p-2$ RNAi, and RNAi in mutants of the steroid signalling pathway revealed that DAF-16 acts by inhibition of DAF-9 and DAF-12. In conclusion, our study demonstrates survival reducing activities caused by the functional loss of ZMP-2 in $C$. elegans. Those effects are mediated by the transport of oxidized cholesterol adducts which then trigger the inhibition of DAF-9 and DAF-12 through the activation of DAF-16.
\end{abstract}

M. Fischer - E. Fitzenberger · R. Kull · M. Boll ·

U. Wenzel $(\square)$

Molecular Nutrition Research, Interdisciplinary Research

Centre, Justus-Liebig-University of Giessen, Heinrich-Buff-Ring

26-32, 35392 Giessen, Germany

e-mail: uwe.wenzel@ernaehrung.uni-giessen.de
Keywords Zinc matrix metalloproteinase $\cdot$ Stress resistance $\cdot$ Steroid signalling - Foxo transcription factor * Cholesterol absorption · Apolipoprotein B

\section{Introduction}

In vertebrates matrix metalloproteinases represent a class of over 26 zinc-dependent enzymes which share a similar structure to each other and are capable of degrading almost every component of the extracellular matrix (Page-McCaw et al. 2007; Hernández-Pérez and Mahalingam 2012; Vu and Werb 2000). Those zinc matrix metalloproteinases are essentially involved in remodelling of the extracellular matrix in both physiological and pathological processes (Siefert and Sarkar 2012). As an example, matrix metalloproteinases (MMPs) are crucial for the generation or regeneration of arteries but their dysregulated activities may also be implicated in the pathogenesis of vascular diseases such as atherosclerosis (Siefert and Sarkar 2012). Interestingly, studies have shown that oxidized low-density lipoproteins and their receptor mediate the up-regulation of vascular factors associated with progression of atherosclerosis, such as MMP-9 (Lund et al. 2011). However, by the progressive accumulation and aggregation of LDL particles in the extracellular matrix, they become targets for oxidative but also enzymatic modifications (Badimon et al. 2011). It is interesting to note in this context that in mammalian keratinocytes, reactive oxygen species (ROS) mediate the effects of heat on tissue destruction and that the expression of several MMPs was induced contributing to tissue regeneration (Shin et al. 2008). Overall this suggests that complex interactions between LDL particles, redox metabolism and MMPs exist.

The nematode Caenorhabditis elegans has been described as an appropriate model to investigate the pathways of 
lipid storage, transport and signalling (Branicky et al. 2010). In the nematode homologues of apolipoprotein B are expressed in terms of vitellogenins (VITs), a class of egg yolk proteins in invertebrates but also in egg-laying vertebrates (Baker 1988). Since C. elegans is auxotroph for sterols (Chitwood and Lusby 1991), one important function of VITs is the provision of sterols to the organism (Matyash et al. 2001). Accordingly, cholesterol must be absorbed from ingested food in the intestine and later into the oocytes. Besides the VITs, transport at both loci requires the accomplishment of receptor-mediated endocytosis through RME-2 (Matyash et al. 2001; Grant and Hirsh 1999). RME2 is a member of the LDL receptor superfamily (Grant and Hirsh 1999), and accordingly, VITs and their receptor may be regarded as evolutionary ancestors of LDL and its receptor in higher organisms (Matyash et al. 2001).

According to homology screening, C. elegans possesses six genes encoding zinc matrix metalloproteinases (ZMPs) (Wada et al. 1998). Recombinant C. elegans ZMPs have been shown to cleave a MMP peptide substrate and this activity was inhibited by human tissue inhibitor of MMP, suggesting that the catalytic sites of $C$. elegans ZMPs and human MMPs are structurally closely related (Wada et al. 1998). Investigating the effects of all six C. elegans ZMPs in vivo, we found in a previous study that only RNAinterference (RNAi) for zmp-2 (H19M22.3) resulted in nematodes with moulting defects indicating its role in extracellular matrix degradation during ecdysis (Altincicek et al. 2010). Moreover, knockdown of zmp-2 was associated with reduced survival under heat stress or during infection with the nematode pathogenic bacterium Photorhabdus luminescens (Altincicek et al. 2010). In hermaphroditic adults, the strongest expression of zmp-2 was detectable in the two spermathecae and spermathecaluterine valves but weaker fluorescence was observed in a zmp-2 promoter::gfp transgenic strain along the intestinal tract (Altincicek et al. 2010).

In the present study, we investigated in $C$. elegans the impact of RNAi for vit- 6 on the reduction of survival caused by zmp-2 RNAi under heat stress.

\section{Materials and methods}

Strains and maintenance

Caenorhabditis elegans strains were maintained on nematode growth medium (NGM) agar plates seeded with E. coli $\mathrm{OP} 50$ at $20{ }^{\circ} \mathrm{C}$ according to standard protocols (Brenner 1974). Caenorhabditis elegans strains wild-type N2, variation Bristol, the mutants DR2281 daf-9(m540), AA1 daf-12(rh257), CF1038 daf-16(mu86), DH1390 rme2(b1008), the transgenic strain TJ356 zIs356 Is[daf- 16::daf-16-gfp; rol-6] and E. coli OP50 were obtained from the C. elegans Genetics Center (University of Minnesota, MN, US). Methods such as freezing nematodes and obtaining synchronous populations using a bleaching method with hypochlorite treatment of egg-laying adults were performed according to standard protocols. RNAi clones were obtained from MRC GeneService Ltd (Cambridge, UK) and included a negative control (L4440), rme2 (T11F8.3), daf-9 (T13C5.1), daf-12 (F11A1.3), daf-16 (R13H8.1), sir-2.1 (R11A8.4), ftt-2 (F52D10.3) and par-5 (M117.2). The RNAi clone of vit-6 (K07H8.6) was constructed using standard cloning techniques as described elsewhere (Fischer et al. 2012).

\section{RNAi experiments}

RNAi experiments were performed in liquid cultures as previously described (Lehner et al. 2006; Timmons et al. 2001; Kamath et al. 2001). In brief, expression of gene-specific dsRNA inside the bacterial RNAi feeding strain E. coli HT115 was induced with $1 \mathrm{mM}$ isopropyl- $\beta$-d-thiogalactopyranoside (IPTG) for $1 \mathrm{~h}$ at $37{ }^{\circ} \mathrm{C}$ to trigger interference. Subsequently, bacterial cells were washed and resuspended in NGM. A volume of $40 \mu \mathrm{l}$ of this suspension was dispensed in each well of a 96-well plate (Greiner Bio-One, Frickenhausen, Germany), to which 10-15 synchronized L1 larvae were added. In general, L1 larvae reached the adult stage within 3 days of incubation with agitation at $20{ }^{\circ} \mathrm{C}$.

\section{Survival analysis at $37{ }^{\circ} \mathrm{C}$}

Nematode survival was determined using a microplate thermotolerance assay as described (Gill et al. 2003). In brief, nematodes were washed off the wells with M9-buffer into $15 \mathrm{ml}$ tubes followed by additional three washing steps. In each well of a black 384-well low-volume microtitre plate (Greiner Bio-One, Frickenhausen, Germany), $6.5 \mu \mathrm{l}$ M9-buffer/Tween 20 (1\% v/v) solution was added. Subsequently, one nematode was dispensed in $1 \mu \mathrm{l}$ M9 buffer under a stereomicroscope (Breukhoven Microscope Systems) into each well and mixed with $7.5 \mu \mathrm{l} \mathrm{SY-}$ TOX green (final concentration $1 \mu \mathrm{M}$; Life Technologies, Karlsruhe, Germany). To prevent water evaporation, the plates were sealed with Rotilabo sealing film and covered with a lid (Greiner Bio-One, Frickenhausen, Germany). Heat shock $\left(37^{\circ} \mathrm{C}\right)$ was induced and fluorescence was measured with the Fluoroskan Ascent fluorometer (Thermo Labsystems, Bonn, Germany) every $30 \mathrm{~min}$. To detect SYTOX green fluorescence, excitation wavelength was set at $485 \mathrm{~nm}$ and emission was measured at $538 \mathrm{~nm}$. All experiments determining the lifespan of nematodes at $37^{\circ} \mathrm{C}$ were done at least thrice and representative survival curves are shown in the figures. 
Quantitative real-time PCR

Total RNA was extracted from 10,000 worms using Trizol (Life Technologies). One-step real-time PCRs were performed in triplicate using $1 \mu \mathrm{l}$ of RNA template, Brilliant II SYBR Green QRT-PCR Mastermix-Kit (Stratagene Products, Waldbronn, Germany) and appropriate primers in a $\mathrm{CFX}^{\mathrm{TM}}$ Real-Time PCR Detection System (BioRad, München, Germany). Cycling conditions were $1 \times[15 \mathrm{~min}$ $\left.50{ }^{\circ} \mathrm{C}\right], 1 \times\left[10 \min 95^{\circ} \mathrm{C}\right], 40 \times\left[30 \mathrm{~s} 95^{\circ} \mathrm{C}, 15 \mathrm{~s} 53^{\circ} \mathrm{C}\right.$, $\left.30 \mathrm{~s} 60^{\circ} \mathrm{C}\right], 1 \times\left[1 \min 95^{\circ} \mathrm{C}\right], 1 \times\left[30 \mathrm{~s} 53^{\circ} \mathrm{C}\right]$ and $1 \times\left[1 \mathrm{~min} 95^{\circ} \mathrm{C}\right]$. Changes in the target gene expression were calculated according to Pfaffl (2001) using equation $2^{-}$ $\Delta \Delta \mathrm{CT}$. For each sample, the fold change in the target gene was normalized to $18 \mathrm{~S}$ rRNA and relative to the control expression. For determination of RNAi efficiency, unique primer pairs (Eurofins MWG Operon, Ebersberg, Germany) recognizing only cDNA derived from endogenous mRNA were designed to avoid cross-reaction with genomic DNA and bacterially generated dsRNA and were as follows: $18 \mathrm{~S}$ $r R N A$-fw 5'-ATG GTT GCA AAG CTG AAA CT-3', $18 S$ $r R N A$-rev $5^{\prime}$-TCC CGT GTT GAG TCA AAT TA-3'; $z m p-2-$ fw 5'-GCT CAG ATT AGA AGA CGA AG-3', zmp-2-rev 5'GAA CTT CAC TTG GAA GAA CA-3'; vit-6-fw 5'-ACC CCA TGC TAC TCC GTT CTC-3', vit-6-rev 5'-CAA CCT TGA CGT TCT TCC ACT CC-3'; daf-9-fw 5'-TTC TGT CTA GCG TTC TCG-3', daf-9-rev 5'-CCA AGA ACT TTG GCT TAT GT-3'; $d a f-12$-fw 5'-CAA CGT GGA TGA TAT GTT TG-3', daf-12-rev 5'-GAA GAA ACC GAA GAA CTC TA-3'; $d a f-16$-fw 5'-CGG AAG GCT TAA ACT CAA TG3'; daf-16-rev 5'-GAC GGA TCG AGT TCT TCC AT-3'.

Relative mRNA levels in nematodes cultured on RNAi bacteria were significantly lower $(p<0.01)$ than mRNA levels of the control that was fed L4440 and were below 0.25 for all genes tested.

\section{Western blot}

For detection of VIT-6 at the protein level, a YP88 rat-antivit-6 polyclonal antibody (kindly provided by Prof. Thomas Blumenthal, University of Colorado) at a 1:5,000 dilution was used and $\beta$-actin, as a loading control, was detected with a rabbit-anti-actin polyclonal antibody (Sigma-Aldrich, Hamburg, Germany) at 1:500. As secondary antibodies goatanti-rat IgG polyclonal (Dianova, Hamburg, Germany) at 1:5,000, and goat-anti-rabbit IgG polyclonal (Santa Cruz, Heidelberg, Germany) at 1:500, respectively, were used as horseradish peroxidase (HRP) conjugate. Groups of 10,000 nematodes were treated with lysis buffer (saccharose $0.8 \mathrm{M}$, EDTA $1 \mathrm{mM}$, Tris-HCL $10 \mathrm{mM}$, PMSF $0.5 \mathrm{mM}$ ) and twice freeze-thawed at $-80{ }^{\circ} \mathrm{C}$ and room temperature, respectively. Lysates were separated on 8.5\% SDS-PAGE gels and proteins were transferred to a polyvinylidene fluoride
(PVDF) membrane (Millipore, Schwalbach, Germany) for $1 \mathrm{~h}$ at $1.0 \mathrm{~mA} / \mathrm{cm}^{2}$ membrane area. The blots were preblocked in $15 \mathrm{ml}$ of $1 \%$ milk in TBS/0.05\% Tween 20 and probed with antibodies diluted in blocking solution for $1 \mathrm{~h}$. Following $2 \times 5$ min washes with TBS-T and $2 \times 5$ min TBS, and incubation in enhanced chemiluminescence (ECL) solution, the radiographic film was exposed to detect the immunoreactive bands. Band intensities were quantified using ImageJ (NIH).

\section{Detection of $\mathrm{H}_{2} \mathrm{O}_{2}$}

Detection of $\mathrm{H}_{2} \mathrm{O}_{2}$ was done by the use of the $N$-acetyl-3,7dihydroxyphenoxazine (Amplex ${ }^{\circledR}$ Ultra Red, SigmaAldrich, Hamburg, Germany) assay. In the presence of $\mathrm{H}_{2} \mathrm{O}_{2}$, Amplex ${ }^{\circledR}$ Ultra Red is oxidized by horseradish peroxidase (HRP) to a red-fluorescent oxidation product (Mohanty et al. 1997). To assess the $\mathrm{H}_{2} \mathrm{O}_{2}$ production from C. elegans, 700 young adult worms per condition were washed four times with $0.25 \mathrm{M} \mathrm{Na}_{2} \mathrm{HPO}_{4}$. Approximately 100 nematodes per $50 \mu \mathrm{l}$ were transferred into flat-bottomed 96-well plates (Greiner Bio-One, Frickenhausen, Germany) and subsequently $50 \mu$ Amplex ${ }^{\circledR}$ Ultra Red reaction buffer solution $\left(50 \mu \mathrm{l} 10 \mathrm{mM}\right.$ Amplex ${ }^{\circledR}$ Ultra Red, $100 \mu \mathrm{l} \mathrm{HRP;}$ $10 \mathrm{U} / \mathrm{ml}, 4.85 \mathrm{ml} 0.25 \mathrm{M} \mathrm{Na}_{2} \mathrm{HPO}_{4}$ ) was added to each well. After 3-h incubation, fluorescence was measured by the use of the microplate reader with excitation at $550 \mathrm{~nm}$ and fluorescence emission detection at $592 \mathrm{~nm}$, respectively.

\section{OxyBlot}

The Millipore OxyBlot ${ }^{\mathrm{TM}}$ Protein Oxidation Detection Kit was used for immunoblot detection of carbonylated proteins. Therefore, groups of 10,000 nematodes were treated with lysis buffer $(0.8 \mathrm{M}$ sucrose, $1 \mathrm{mM}$ EDTA, $10 \mathrm{mM}$ Tris-HCL, $0.5 \mathrm{mM}$ PMSF), twice freeze-thawed at $-80{ }^{\circ} \mathrm{C}$ and room temperature, respectively, and subsequently sonicated on ice. The concentration of isolated proteins was adjusted to $1.5 \mathrm{mg} / \mathrm{ml}$ before derivatization was performed according to the instruction manual, using $5 \mu \mathrm{l}$ protein sample. Derivatized proteins were separated by $8.5 \%$ SDSPAGE and blotted onto a PVDF membrane (1 h, $1.0 \mathrm{~mA} /$ $\mathrm{cm}^{2}$ ). The membrane was blocked with $1 \%$ milk in TBS/ $0.05 \%$ Tween ${ }^{\circledR} 20$ and probed with antibodies as described in the instruction manual of the OxyBlot kit. $\beta$-Actin from non-derivatized samples was detected as a loading control for the normalization of intensities of the bands between the different treatments by the use of a horseradish peroxidaseconjugated goat-anti-rabbit IgG (Santa Cruz, Heidelberg, Germany). For chemiluminescence detection, the membrane was incubated for $1 \mathrm{~min}$ with freshly prepared enhanced chemiluminescent solution (1 M Tris $\mathrm{pH} 8.5$, $90 \mathrm{mM}$ p-coumaric acid, $250 \mathrm{mM}$ luminol, $0.01 \% \mathrm{H}_{2} \mathrm{O}_{2}$ ). 
An autoradiography film was exposed for $5 \mathrm{~min}$ to detect immunoreactive bands. Band intensities were quantified using ImageJ (NIH).

\section{Microscopic analyses}

To visualize ROS stress located in mitochondria, worms were grown in liquid culture and treated for $24 \mathrm{~h}$ at $20^{\circ} \mathrm{C}$ in the young adult stage with $0.5 \mu \mathrm{g} / \mathrm{ml}$ MitoTracker $\mathrm{CM}-\mathrm{H}_{2} \mathrm{XRos}$ (Life Technologies). CM- $\mathrm{H}_{2} \mathrm{XRos}$ produces a ROS-specific staining of mitochondria within cells that is amenable to both qualitative and quantitative measurements of mitochondrial production of $\mathrm{H}_{2} \mathrm{O}_{2}$ (Degli Esposti 2002). It does not get fluorescent until it enters an actively respiring cell, where it is oxidized predominantly by reactions involving hydrogen peroxide production (Degli Esposti 2002). According to its positive charge acquired on oxidation by intracellular ROS, the probe covalently binds to mitochondrial proteins and thus produces a permanent organelle-specific stain (Degli Esposti 2002). The MitoTracker fluorescence was measured at $580 \mathrm{~nm}$ after excitation at $530-585 \mathrm{~nm}$ by the use of an Axioskop 2 epifluorescence microscope (Zeiss, Jena). The microscopic images were generated as 24-bit-jpeg format, which were quantified by using ImageJ (NIH).

Dehydroergosterol (Sigma, Deisehofen, Germany) uptake was measured using the Axioskop2 as described for MitoTracker staining. Therefore, L1 larvae were grown as described for RNAi experiments in the presence of $100 \mu \mathrm{M}$ dehydroergosterol. Fluorescence was measured after excitations at $340-380 \mathrm{~nm}$ and emissions at $425 \mathrm{~nm}$ and quantified by using ImageJ (NIH).

\section{Calculations and statistics}

Statistical analyses were performed with GraphPad Prism 5.0 software (GraphPad, La Jolla, CA, USA). Log-rank test was performed to compare survival rates under heat stress with $p$ values $<0.05$ being significant. Kaplan-Meier survival curves are shown for lifespan experiments, which all have been repeated more than once with similar effects. For group comparisons, $t$ test or analysis of variance (ANOVA) was performed. Following ANOVA, differences between groups were determined by Bonferroni-Holm multiple comparison test. Results are presented as mean $\pm \mathrm{SD}$.

\section{Results}

Vit-6 RNAi blocks the reduction of thermotolerance caused by zmp-2 RNAi

Since ZMPs through alteration of extracellular proteins affect several pathologies associated with reduced stress resistance, we tested here its influence on heat-stress resistance in the model organism $C$. elegans. Knockdown of $z m p-2$ was associated with a significant reduction of survival of wild-type nematodes at $37{ }^{\circ} \mathrm{C}$ (Fig. 1a). Suggesting that lipoproteins could be a target for ZMP-2, we assessed in how far uptake of lipoproteins into cells plays a role for the $z m p-2$ RNAi-induced reduction of thermotolerance. Reducing the transcript levels of vit- 6 by RNAi was found to be associated with a complete rescue of the lifespan reducing activity exerted by zmp-2 RNAi (Fig. 1a), suggesting that indeed $z m p-2$ RNAi alters lipoproteins extracellular. Knockdown of rme-2, encoding the VIT-receptor, likewise to the knockdown of vit-6, completely abolished the lifespan reduction caused by knockdown of zmp-2 (Fig. 1b).

Vit-6 RNAi increases the ROS burden inside and outside of $C$. elegans

Since VIT-6 besides its transport-relevant functions displays also antioxidative functions, we were interested in how far its knockdown affects intra- and extracellular ROS levels. RNAi for $z m p-2$ resulted in an increase of MitoTracker $\mathrm{CM}-\mathrm{H}_{2}$ XRos fluorescence, indicating increased intracellular ROS (Fig. 2a), enhanced protein carbonyls as a measure of protein oxidation (Fig. 2b), and also an increase of ROS in the extracellular compartment as measured using Amplex Red (Fig. 2c). Although not significantly, all three parameters were further enhanced by additional vit-6 RNAi (Fig. 2a-c), indicating that the unaltered lifespan versus the control under vit-6 and zmp-2 double RNAi occurs in spite of increased ROS stress. To substantiate that extracellular oxidized lipoproteins are responsible for the thermotolerance reducing effects of $z m p-2$ RNAi, the ROS scavenger ascorbic acid was applied. This reduced the ROS levels in the extracellular mileu of $z m p-2$ RNAi-treated nematodes to the levels of the control (Fig. 2c) and moreover prevented any effects on survival at $37^{\circ} \mathrm{C}$ by $z m p-2$ RNAi (Fig. 2d). The importance of increasingly accumulating oxidatively modified lipoprotein constituents outside the cells and their transport into the cells as the basis for the reduced thermotolerance caused by zmp2 RNAi is further substantiated by the reduced expression of vit-6 at the transcript level (Fig. 3a) and also at the protein level (Fig. 3b) when zmp-2 was knocked down. Since under these conditions reduction of survival is fully evident, we suggested that a further restriction of oxidized lipoprotein uptake by vit-6 RNAi will provide protection. As shown, vit-6 RNAi indeed led to a significant lower uptake of the cholesterol homologue DHE than zmp-2 RNAi (Fig. 3c). 
Fig. 1 Cholesterol transport is essential for the reduction of survival under heat stress by $z m p-2$ knockdown. RNAi versus vit-6 completely rescues the lifespan reduction which is due to zmp-2 RNAi in N2 wild-type nematodes (a). The functional loss of a receptor for receptormediated endocytosis in the rme-2 mutant prevented the toxicity mediated by zmp-2 RNAi in N2 completely (b)
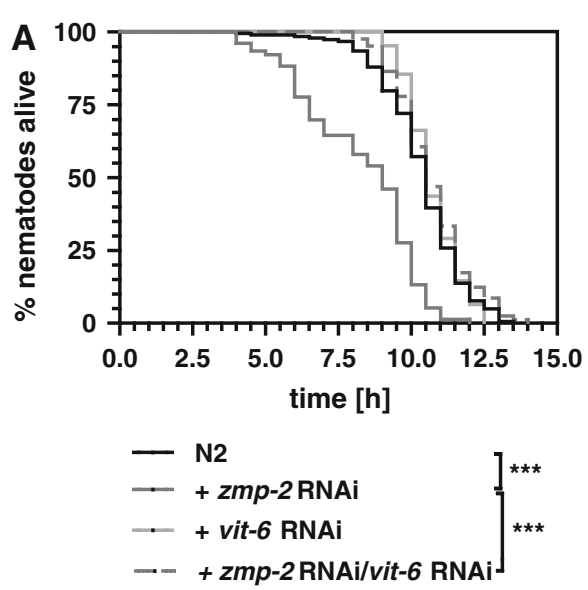

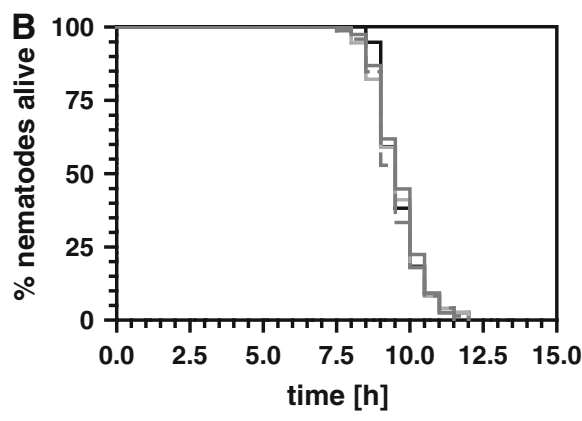

- rme-2

-- + zmp-2 RNAi

-+ vit-6 RNAi

- + zmp-2 RNAi/vit-6 RNAi
A
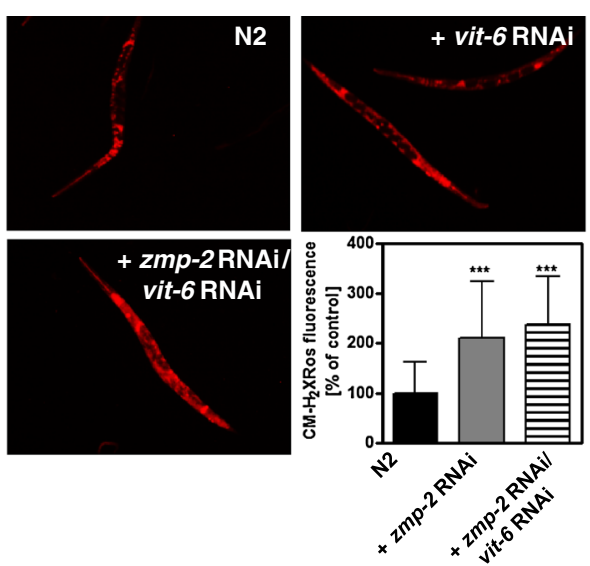

C.

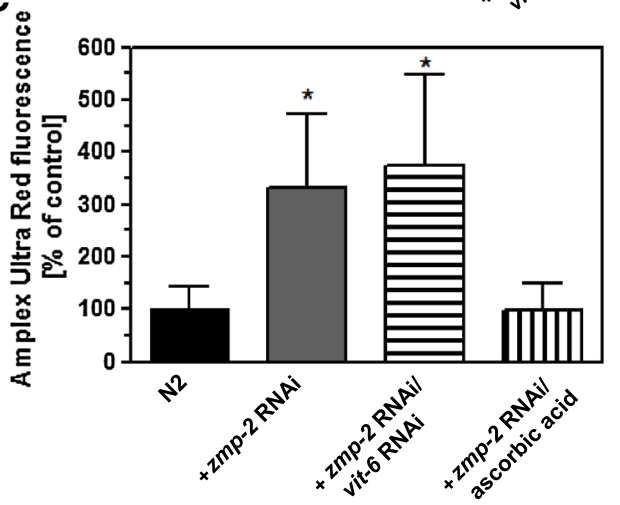

B

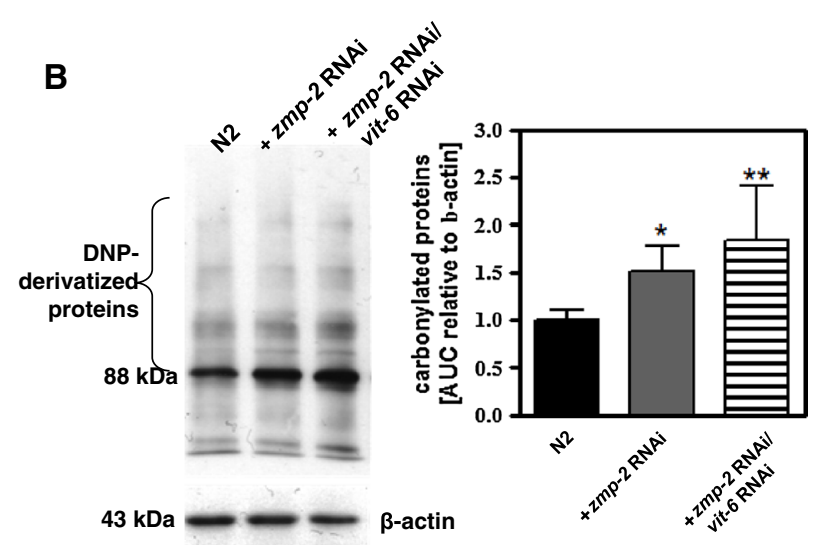

D

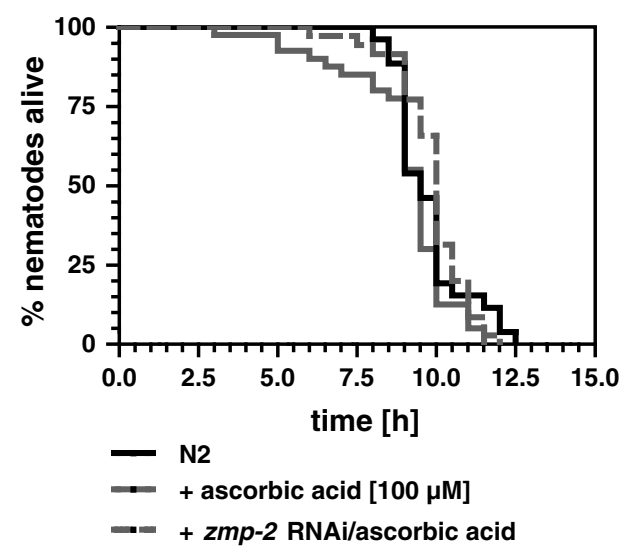

nematodes treated with either $z m p-2$ RNAi or $z m p-2$ plus vit-6 RNAi revealed an increased ROS secretion in response to both RNAi treatments versus the control (c). Using the ROS scavenger ascorbic acid at a concentration of $100 \mu \mathrm{M}$ was able to prevent the increase in extracellular ROS due to zmp-2 RNAi (c) and also the reduction of thermotolerance caused by zmp-2 RNAi in wild-type nematodes (d). $* p<0.05 ; * * p<0.01 ; * p<0.001$ versus the $\mathrm{N} 2$ control 
Fig. 3 Zmp-2 RNAi decreases vit-6 expression and DHE uptake. Real-time quantitative PCR (a) and Western blot analysis with $\beta$-actin as a loading control (b) show the significantly decreased vit- 6 expression at the mRNA and protein level, respectively, in nematodes treated with $z m p-2$ RNAi. Uptake of dehydroergosterol was measured according to its fluorescent properties using fluorescence microscopy (c) and quantified using ImageJ (c, right lower panel). $* * p<0.01$; $* * * p<0.001$ versus the $\mathrm{N} 2$ vector control; ${ }^{\# \#} p<0.01$ versus zmp-2 RNAi-treated nematodes

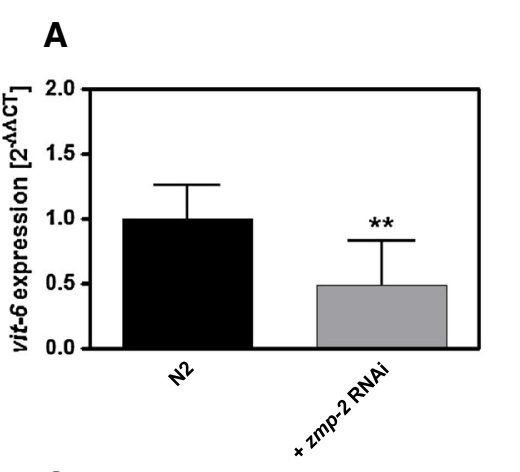

\section{C}
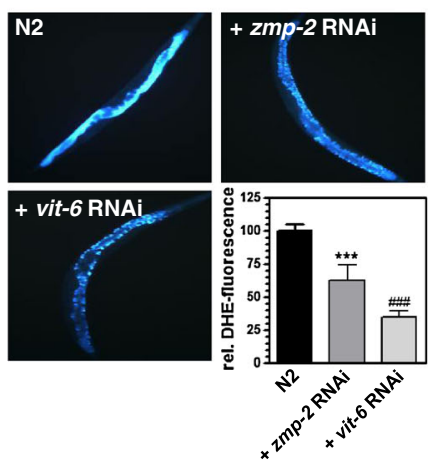

B

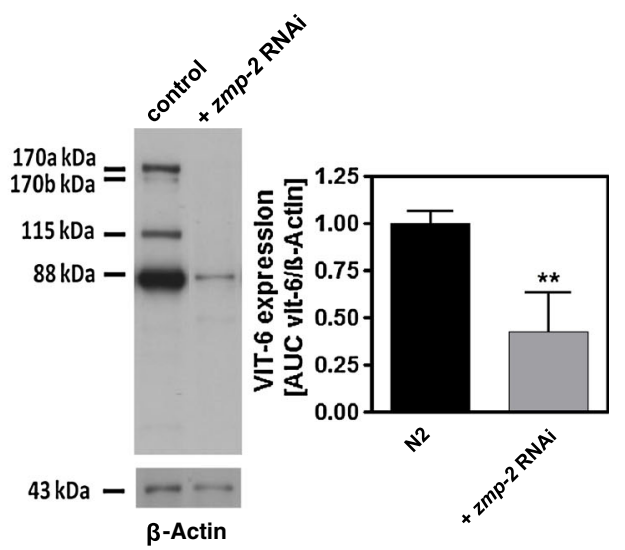

Fig. 4 DAF-16 mediates the detrimental effects of $z m p-2$ RNAi. When daf-16 was knocked down by RNAi, $z m p-2$ RNAi was no longer able to reduce the survival of wild-type C. elegans at $37^{\circ} \mathrm{C}$ (a). DAF-16 was exclusively localized in the nuclei after $z m p-2$ RNAi as was evidenced using the transgenic strain TJ356 expressing DAF$16::$ GFP (b)

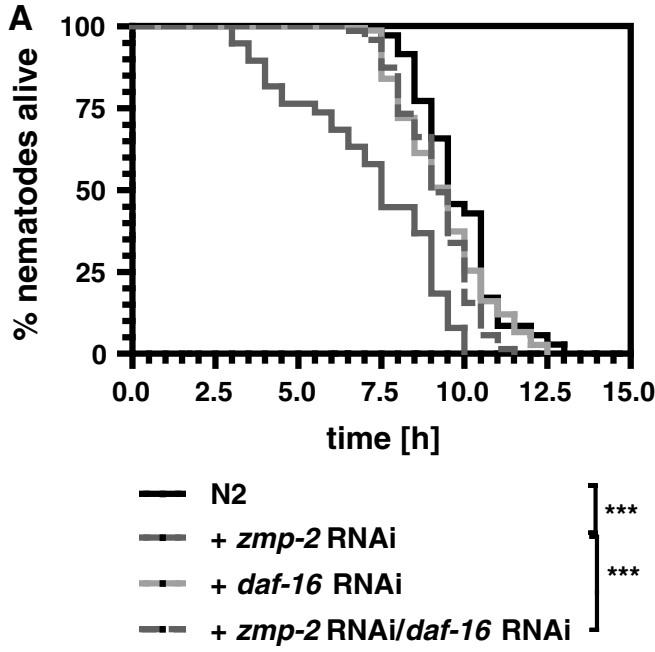

B
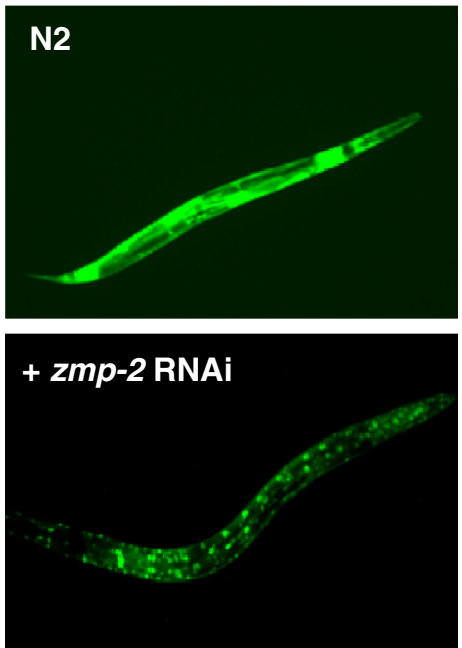

Zmp-2 RNAi reduces survival under heat stress depending on DAF-16 which inhibits DAF-9 and DAF-12

The foxo transcription factor DAF-16 has been shown in many studies to be pivotal for resistance against various kinds of stress. It was astonishing, therefore, that the knockdown of daf-16 was able to reverse the thermotolerance reduction induced by zmp-2 RNAi (Fig. 4a). Since it has been demonstrated that SIR-2.1 and also the 14-3-3 proteins FTT-2 and PAR-5 can function as transcriptional co-activators of DAF-16 (Berdichevsky et al. 2006; Berdichevsky and Guarente 2006), also their impact on survival as affected by $z m p-2$ RNAi was studied. It was found that likewise to the knockdown of daf-16 also the knockdown of each of the three co-activators prevented any thermotolerance diminishing effect of $z m p-2$ RNAi (Table 1). Moreover, in zmp-2 RNAi-treated nematodes, DAF-16 was found to be exclusively localized to the nuclei (Fig. 4b). 
Table 1 RNAi for $s i r-2.1, f t$ - 2 or par-5 prevent the reduction of survival under heat stress caused by zmp-2 RNAi

\begin{tabular}{llll}
\hline $\begin{array}{l}\text { RNAi } \\
\text { treatment }\end{array}$ & $\begin{array}{l}\text { Average } \\
\text { lifespan } \pm \mathrm{SD}[\mathrm{h}]\end{array}$ & $\begin{array}{l}\text { Number of } \\
\text { nematodes }\end{array}$ & Significance \\
\hline vector control & $9.8 \pm 1.3$ & 70 & \\
zmp-2 & $7.2 \pm 2.3$ & 76 & $* * *$ \\
sir-2.1 & $9.3 \pm 2.0$ & 76 & \\
zmp-2/sir-2.1 & $9.4 \pm 1.9$ & 73 & $\#_{* * *}$ \\
ftt-2 & $9.3 \pm 1.4$ & 75 & \\
zmp-2/ftt-2 & $9.1 \pm 1.1$ & 73 & \#*** \\
par-5 & $9.0 \pm 1.0$ & 76 & $*$ \\
zmp-2/par-5 & $8.9 \pm 1.1$ & 77 & \#*** \\
\hline
\end{tabular}

$* p<0.05$ versus control; $* * * p<0.001$ versus control; \#*** $p<0.001$ versus $z m p-2$ RNAi

In previous studies, we have found that stress resistance against nematode pathogenic bacteria is dependent on the steroid signalling pathway (Fischer et al. 2013) and especially its members DAF-9 and DAF-12 (Rottiers et al. 2006; Gerisch et al. 2001). As a matter of fact, mutants for daf-9 (Fig. 5a) or daf-12 (Fig. 5b) were hypersensitive versus the knockdown of zmp-2. That DAF-16 acts through the inhibition of DAF-9 and DAF-12 was evidenced by the knockdown of daf-16 in either daf-9 or daf-12 mutants. Both mutants no longer responded under zmp-2 RNAi to daf-16 RNAi with an extension of survival at $37{ }^{\circ} \mathrm{C}$ (Fig. 5c, d).

A basic supply of cholesterol is mandatory for activation of steroid signalling and the increase in thermotolerance under $z m p-2 \mathrm{RNAi}$

The finding that DAF-9 and DAF-12 exerted protective effects to zmp-2 RNAi (Fig. 5a, b) was unexpected, based on the increased nematode survival also under $z m p-2$ RNAi when delivery of their activator cholesterol was reduced by vit-6 or rme-2 RNAi (Fig. 1). As a matter of fact, however, in both daf-9 and daf-12 mutants vit-6 RNAi exerted no effect on the reduction in thermotolerance caused by zmp-2 RNAi (Fig. 6a, b). We therefore suggested that a basic supply of cholesterol is necessary and possible under vit-6 RNAi in order to enable the activation of the steroid signalling pathway and to prolong the lifespan under zmp-2 knockdown. This hypothesis is supported by the fact that in wild-type nematodes residual intestinal uptake of the cholesterol homologue dehydroergosterol in the presence of vit-6 RNAi is evident (Fig. 3c). Moreover, reducing the cholesterol concentration in the feeding media to $2.5 \mu \mathrm{g} / \mathrm{ml}$ prevented vit-6 RNAi to exert its reverting effects on survival reduction in wild-type nematodes which was due to zmp-2 RNAi (Fig. 6c). Finally, a daf-16 deletion mutant
Fig. 5 DAF-9 and DAF-12 are required for resistance versus zmp-2 RNAi-mediated toxicity. In nematodes mutant for daf-9 (a) or daf-12 (b) zmp-2 RNAi reduced the thermotolerance to a significant higher extent than in wild-type nematodes. Daf-16 RNAi performed in both mutant strains revealed that the knockdown of the foxo transcription factor is no longer able to prevent the survival reduction as caused by zmp-2 RNAi under subsequent heat stress (c, d). Statistical analysis is only shown for groups treated with $z m p-2$ RNAi
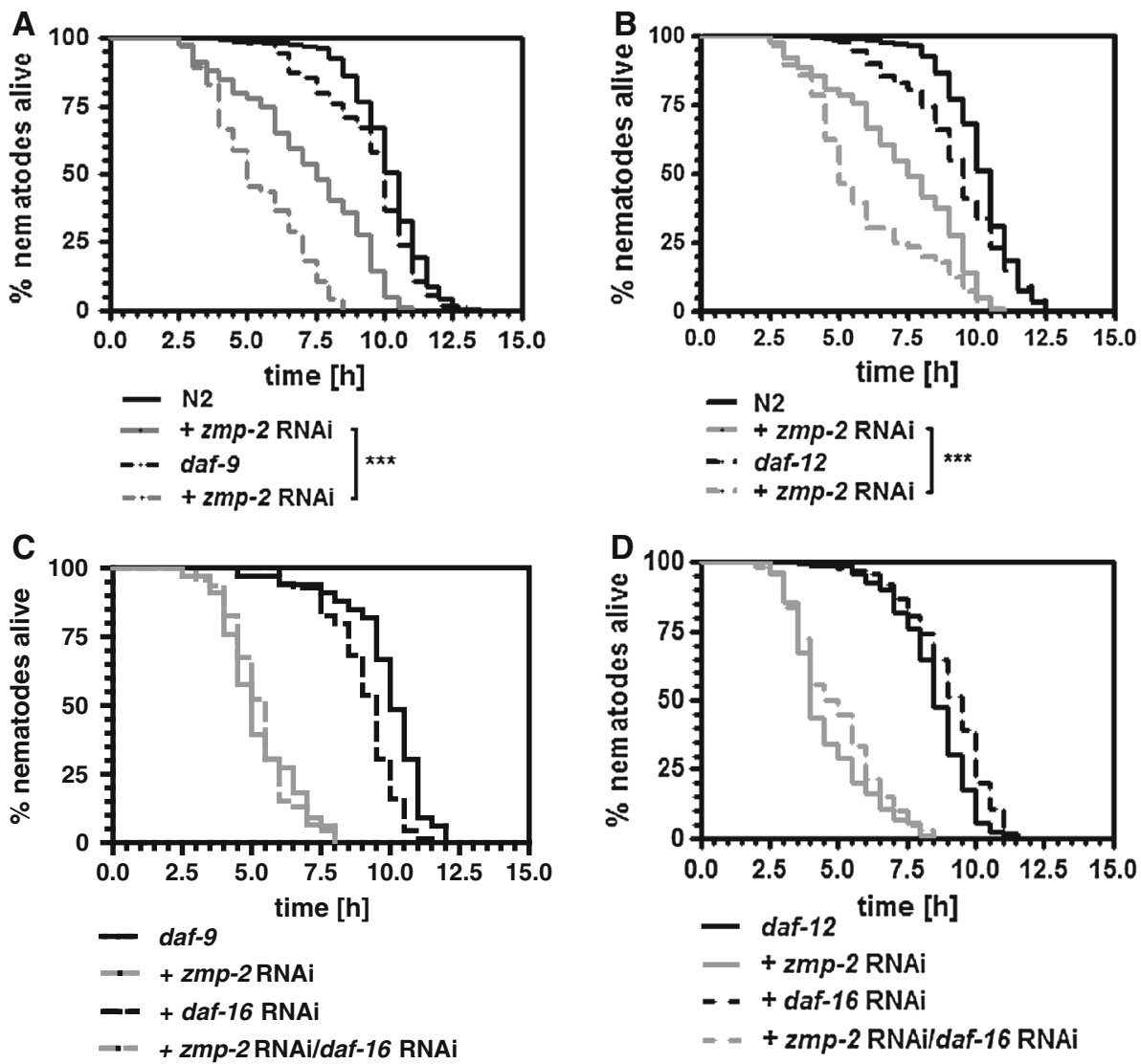
Fig. 6 Vit-6 RNAi needs DAF9 and DAF-12 in order to prevent $z m p-2$ RNAi-induced reduction of thermotolerance. In daf-9 (a) and daf-12 (b) mutant animals, the knockdown of vit-6 was no longer effective in reverting the $z m p-2$ RNAiinduced reduction in survival. The suggestion that under vit- 6 RNAi low levels of cholesterol are transported in order to trigger DAF-9, and DAF12 is supported by a lack of survival reverting effect of vit-6 RNAi when $2.5 \mu \mathrm{g}$ cholesterol $/ \mathrm{ml}$ NGM instead of $5 \mu \mathrm{g}$ cholesterol/ml NGM were fed (c). Moreover, in daf-16 mutant nematodes, vit-6 RNAi in zmp-2 RNAi treated nematodes reduced survival, indicating the requirement of minimal cholesterol for the vit-6 RNAimediated survival extension (d). Statistical analysis is only shown for groups treated with zmp-2 RNAi
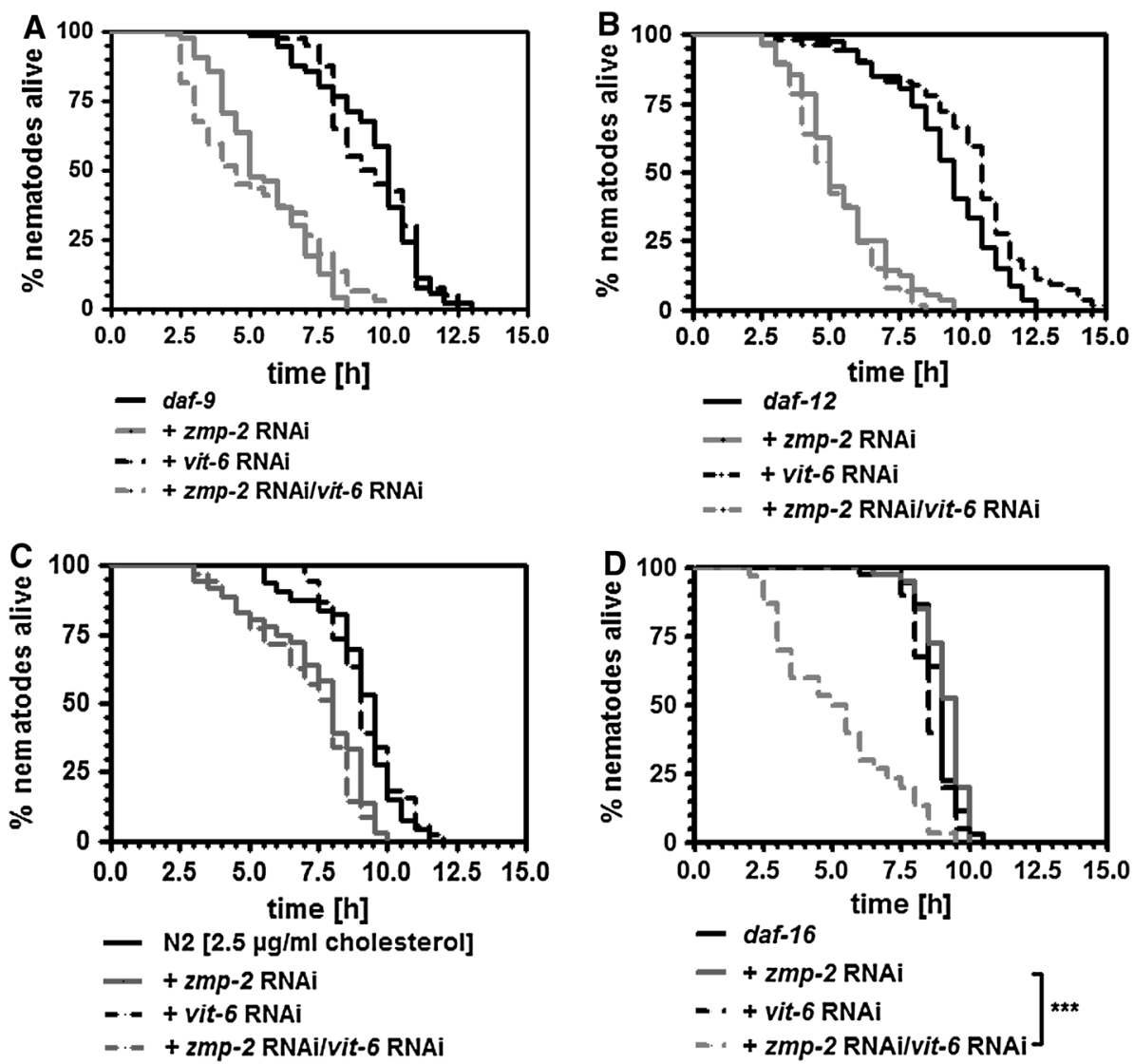

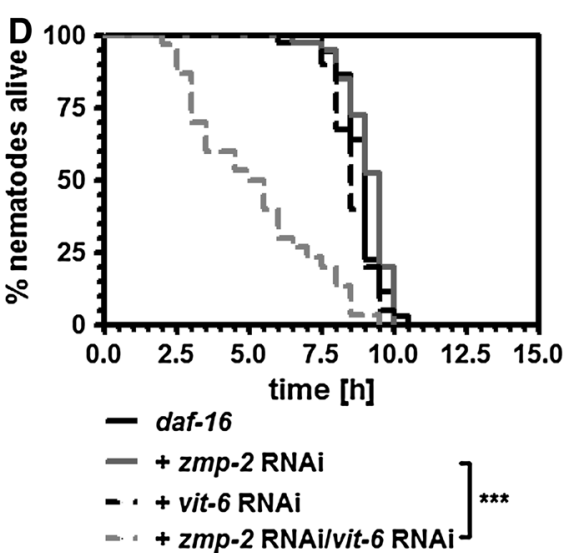

proved to be resistant against zmp-2 RNAi but became hypersensitive when vit- 6 was knocked down in addition (Fig. 6d).

\section{Discussion}

In the present study, we have shown that knockdown of the zinc matrix metalloproteinase zmp-2 in C. elegans significantly reduces the resistance versus heat stress. Those results are in agreement with findings suggesting that zincdependent mechanisms, maybe represented by the activities of MMPs, can be a single mediator in modulating neuroendocrine-thymus interaction in ageing (Mocchegiani et al. 2013). Zmp-2 RNAi caused an increase of intracellular and extracellular ROS, but since reductions of thermotolerance were completely inhibited when transport of lipoproteins was reduced through the knockdown of vit6 , we suggest that transport of oxidized lipoproteins into the cells mediates the effects of zmp-2 knockdown on thermotolerance (Fig. 7). In search of the oxidized compound mediating the reduction in stress resistance, the most plausible explanation would be provided by the oxidation of VIT- 6 itself, since it has been demonstrated to be the most abundant oxidized protein in adult nematodes
(Nakamura et al. 1999). Indeed protein carbonylation of a $88 \mathrm{kDa}$ protein which could represent VIT-6 was increased by zmp-2 RNAi in our studies. Alternative explanations are that the oxidative mileu caused by zmp-2 RNAi oxidizes other apolipoproteins than VIT-6 or that VIT-6 is just the mediator of transport, e.g. for oxysterols, which are a hallmark of a number of age-related diseases in humans, such as atherosclerosis, neurodegenerative diseases or agerelated macular degeneration (Poli et al. 2013). Whatever the stress resistance reducing effector might be, our results make clear that lipoprotein transport essentially mediates the detrimental effects of $z m p-2 \mathrm{RNAi}$, which alternatively could also be prevented by the addition of ascorbic acid. Ascorbic acid reduces the ROS burden at the extracellular site and must act from this compartment since thermotolerance is increased by vit- 6 RNAi instead of increased intracellular ROS levels and protein carbonyls.

It was surprising to note that the reductions of thermotolerance caused by the knockdown of zmp-2 were completely dependent on the presence of the foxo transcription factor DAF-16, which has been described to transcriptionally trigger several stress response mechanisms in association with longevity (McColl et al. 2010). On the other hand, we have previously demonstrated that the prevention of nuclear DAF-16 localization by VIT-6 is 


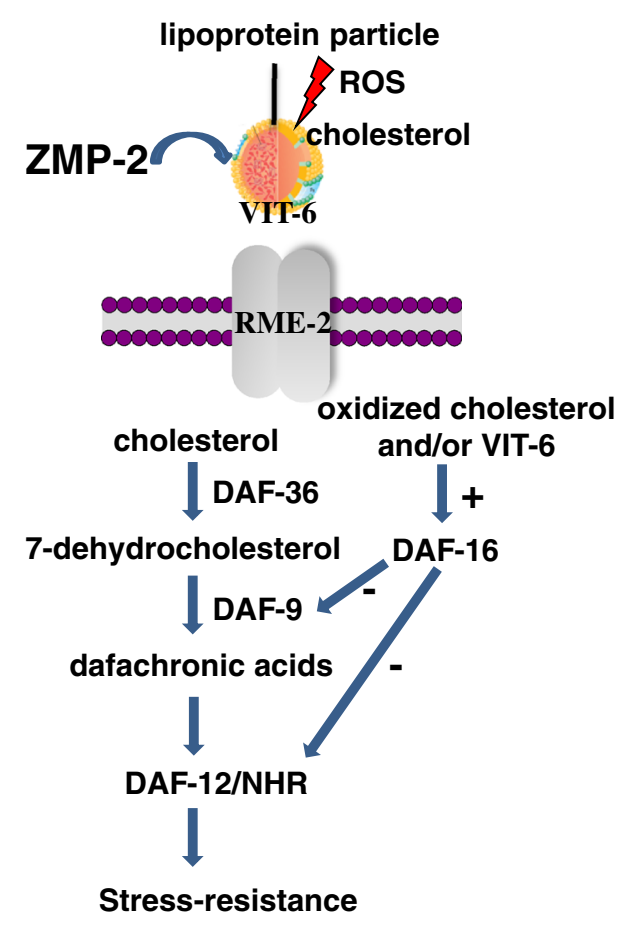

Fig. 7 Concept explaining the effects of the zinc matrix metalloproteinase ZMP-2 on stress resistance in $C$. elegans as impaired by the transport of oxidized cholesterol adducts. Extracellular cholesterol can be exploited by the cells subsequent to its transport requiring VIT-6 and RME-2, in order to trigger the steroid signalling pathway. Extracellular ROS, however, can oxidize cholesterol or lipoproteins before their cellular uptake. Once inside the cell, oxidized cholesterol causes a cellular response which involves DAF-16 and consequently inhibits DAF-9 and DAF-12. Those effects are associated with reduced stress resistance and can be prevented either by reducing the uptake of oxidized cholesterol adducts or by ZMP-2, which is suggested to degrade such cytotoxic products

associated with defence mechanisms against a nematode pathogen (Fischer et al. 2013) demonstrating that enhanced resistance, as achieved, e.g. by the prevention of faulty water homoeostasis (Evans et al. 2008), can also derive from the absence of transcriptional activity of DAF-16. Here, however, it is not that the absence of DAF-16 transcriptional activity provides stress resistance but that its nuclear localization is found to be associated with the survival reductions caused by zmp-2 RNAi. That indeed the $z m p-2$ RNAi effects on thermotolerance are mediated through transcriptional activity of DAF-16 is further stressed by the fact that the knockdown of its transcriptional co-activators sir-2.1, ftt-2 and par-5 (Berdichevsky et al. 2006; Berdichevsky and Guarente 2006) prevented the reduced thermotolerance as caused by $z m p-2$ RNAi in an identical manner as daf-16 RNAi.

Besides the insulin signalling pathway with its key factor DAF-16, the steroid signalling pathway including its key components DAF-9, a cytochrome $\mathrm{P}_{450}$-dependent enzyme forming dafachronic acids, and the nuclear hormone receptor DAF-12, recognizing dafachronic acids as ligands, has been shown to exert significant effects on lifespan and survival (Rottiers et al. 2006; Gerisch et al. 2001). Also in this regard, the picture is not completely clear, given that dafachronic acid supplementation shortened the lifespan of long-lived daf-9 mutants and abolished their stress resistance, whereas they extended the lifespan of germ-line ablated daf-9 mutants, demonstrating that the steroid signalling pathway may regulate $C$. elegans lifespan according to signalling state (Gerisch et al. 2007). In the present study, we demonstrate that both DAF-9 and DAF12 are involved in the protection against the toxicity mediated by zmp-2 RNAi (Fig. 7). Strains mutant for either daf-9 or daf-12 were hypersensitive to $z m p-2$ RNAi. Moreover, in these strains, daf-16 RNAi no longer provided any protection from zmp-2 RNAi, demonstrating that both factors act downstream from DAF-16 and are inhibited by the foxo transcription factor (Fig. 7).

Since cholesterol, however, provides the substrate for steroid signalling, it has to be assumed that a minimal supply of sterols is mandatory in order to enable DAF-9 to generate ligands for DAF-12 and to compensate for the detrimental effects of zmp-2 RNAi (Fig. 7). This hypothesis is substantiated by the results obtained in wild-type nematodes which were fed low cholesterol concentrations because they did not respond with an extended survival when vit-6 was knocked down in zmp-2 RNAi exposed nematodes. Also the daf-16 RNAi-mediated resistance to the reductions of survival by zmp-2 RNAi proved to be dependent on an adequate minimal cholesterol supply since double RNAi for $z m p-2$ and vit- 6 reduced the survival of daf-16 mutants dramatically.

In conclusion, we provide evidence that ZMP-2 in $C$. elegans prevents the accumulation of oxidized lipoproteins containing VIT- 6 which are absorbed into the nematodes by receptor-mediated endocytosis. A lack of ZMP-2 activity leads to the absorption of toxic adducts which then trigger DAF-16 to inhibit DAF-9 and DAF-12 and lead to diminished thermotolerance.

Conflict of interest None.

\section{References}

Altincicek B, Fischer M, Lüersen K, Boll M, Wenzel U, Vilcinskas A (2010) Role of matrix metalloproteinase ZMP-2 in pathogen resistance and development in Caenorhabditis elegans. Dev Comp Immunol 34:1160-1169

Badimon L, Storey RF, Vilahur G (2011) Update on lipids, inflammation and atherothrombosis. Thromb Haemost 105: S34-S42

Baker ME (1988) Is vitellogenin an ancestor of apolipoprotein B-100 of human lowdensity lipoprotein and human lipoprotein lipase? Biochem J 255:1057-1060 
Berdichevsky A, Guarente L (2006) A stress response pathway involving sirtuins, forkheads and 14-3-3 proteins. Cell Cycle 5:2588-2591

Berdichevsky A, Viswanathan M, Horvitz HR, Guarente L (2006) $C$. elegans SIR-2.1 interacts with 14-3-3 proteins to activate DAF16 and extend lifespan. Cell 125:1165-1177

Branicky R, Desjardins D, Liu JL, Hekimi S (2010) Lipid transport and signaling in Caenorhabditis elegans. Dev Dyn 239: $1365-1377$

Brenner S (1974) The genetics of Caenorhabditis elegans. Genet Dev 77:71-94

Chitwood DJ, Lusby WR (1991) Metabolism of plant sterols by nematodes. Lipids 26:619-627

Degli Esposti M (2002) Measuring mitochondrial reactive oxygen species. Methods 26:335-340

Evans EA, Kawli T, Tan MW (2008) Pseudomonas aeruginosa suppresses host immunity by activating the DAF-2 insulin-like signaling pathway in Caenorhabditis elegans. PLoS Pathog 4:1000175

Fischer M, Regitz C, Kahl M, Werthebach M, Boll M, Wenzel U (2012) Phytoestrogens genistein and daidzein affect immunity in the nematode Caenorhabditis elegans via alterations of vitellogenin expression. Mol Nutr Food Res 56:957-965

Fischer M, Regitz C, Kull R, Boll M, Wenzel U (2013) Vitellogenins increase stress resistance of Caenorhabditis elegans after Photorhabdus luminescens infection depending on the steroidsignaling pathway. Microbes Infect 15:569-578

Gerisch B, Weitzel C, Kober-Eisermann C, Rottiers V, Antebi A (2001) A hormonal signaling pathway influencing C. elegans metabolism, reproductive development, and life span. Dev Cell 1:841-851

Gerisch B, Rottiers V, Li D, Motola DL, Cummins CL, Lehrach H, Mangelsdorf DJ, Antebi A (2007) A bile acid-like steroid modulates Caenorhabditis elegans lifespan through nuclear receptor signaling. Proc Natl Acad Sci 104:5014-5019

Gill MS, Olsen A, Sampayo JN, Lithgow GJ (2003) An automated high-throughput assay for survival of the nematode Caenorhabditis elegans. Free Radic Biol Med 35:558-565

Grant B, Hirsh D (1999) Receptor-mediated endocytosis in the Caenorhabditis elegans oocyte. Mol Biol Cell 10:4311-4326

Hernández-Pérez M, Mahalingam M (2012) Matrix metalloproteinases in health and disease: insights from dermatopathology. Am J Dermatopathol 34:565-579

Kamath RS, Martinez-Campos M, Zipperlen P, Fraser AG, Ahringer J (2001) Effectiveness of specific RNA-mediated interference through ingested double-stranded RNA in Caenorhabditis elegans. Genome Biol 2:RESEARCH0002

Lehner B, Tischler J, Fraser AG (2006) RNAi screens in Caenorhabditis elegans in a 96-well liquid format and their application to the systematic identification of genetic interactions. Nat Protoc 1:1617-1620
Lund AK, Lucero J, Harman M, Madden MC, McDonald JD, Seagrave JC, Campen MJ (2011) The oxidized low-density lipoprotein receptor mediates vascular effects of inhaled vehicle emissions. Am J Respir Crit Care Med 184:82-91

Matyash V, Geier C, Henske A, Mukherjee S, Hirsh D, Thiele C, Grant B, Maxfield FR, Kurzchalia TV (2001) Distribution and transport of cholesterol in Caenorhabditis elegans. Mol Biol Cell 12:1725-1736

McColl G, Rogers AN, Alavez S, Hubbard AE, Melov S, Link CD, Bush AI, Kapahi P, Lithgow GJ (2010) Insulin-like signaling determines survival during stress via posttranscriptional mechanisms in C. elegans. Cell Metab 12:260-272

Mocchegiani E, Malavolta M, Costarelli L, Giacconi R, Piacenza F, Lattanzio F, Basso A (2013) Is there a possible single mediator in modulating neuroendocrine-thymus interaction in ageing? Curr Aging Sci 6:99-107

Mohanty JG, Jaffe JS, Schulman ES, Raible DG (1997) A highly sensitive fluorescent micro-assay of $\mathrm{H} 2 \mathrm{O} 2$ release from activated human leukocytes using a dihydroxyphenoxazine derivative. J Immunol Methods 202:133-141

Nakamura A, Yasuda K, Adachi H, Sakurai Y, Ishii N, Goto S (1999) Vitellogenin-6 Is a Major Carbonylated Protein in Aged Nematode, Caenorhabditis elegans. Biochem Biophys Res Commun 264:580-583

Page-McCaw A, Ewald AJ, Werb Z (2007) Matrix metalloproteinases and the regulation of tissue remodelling. Nat Rev Mol Cell Biol 8:221-233

Pfaffl MW (2001) A new mathematical model for relative quantification in real-time T-PCR. Nucleic Acids Res 92:2002-2007

Poli G, Biasi F, Leonarduzzi G (2013) Oxysterols in the pathogenesis of major chronic diseases. Redox Biol 1:125-130

Rottiers V, Motola DL, Gerisch B, Cummins CL, Nishiwaki K, Mangelsdorf DJ, Antebi A (2006) Hormonal control of $C$. elegans dauer formation and life span by a Rieske-like oxygenase. Dev Cell 10:473-482

Shin MH, Moon YJ, Seo JE, Lee Y, Kim KH, Chung JH (2008) Reactive oxygen species produced by NADPH oxidase, xanthine oxidase, and mitochondrial electron transport system mediate heat shock-induced MMP-1 and MMP-9 expression. Free Radic Biol Med 44:635-645

Siefert SA, Sarkar R (2012) Matrix metalloproteinases in vascular physiology and disease. Vascular 20:210-216

Timmons L, Court DL, Fire A (2001) Ingestion of bacterially expressed dsRNAs can produce specific and potent genetic interference in Caenorhabditis elegans. Gene 263:103-112

$\mathrm{Vu} \mathrm{TH}$, Werb Z (2000) Matrix metalloproteinases: effectors of development and normal physiology. Genes Dev 14:2123-2133

Wada K, Sato H, Kinoh H, Kajita M, Yamamoto H, Seiki M (1998) Cloning of three Caenorhabditis elegans genes potentially encoding novel matrix metalloproteinases. Gene 211:57-62 